Trauma Berufskrankh 2007 - 9 [Suppl 2]:S168-S171 DOI 10.1007/s10039-006-1146-7

Online publiziert: 31. Mai 2006

(c) Springer Medizin Verlag 2006
G. O. Hofmann · I. Marintschev ·. Mückley · F. Mendler

Kliniken für Unfall-, Hand- und Wiederherstellungschirurgie, BG-Kliniken

Bergmannstrost, Friedrich-Schiller-Universität Jena, Halle, Saale

\title{
Beckenring- und Azetabulumverletzungen
}

\section{Stellenwert der Navigation}

Für die definitive Versorgung von Beckenringverletzungen und Azetabulumfrakturen sind meist ausgedehnte operative Zugänge erforderlich. Die Exposition der knöchernen Anteile des vorderen Beckenrings erfolgt über den so genannten Pfannenstielzugang und den medialen Teilabschnitt des ilioinguinalen Zugangs (Zugang nach Letournel). Der hintere Beckenring ist durch den ilioinguinalen Zugang in seinem lateralen Teilabschnitt von ventral erreichbar, ebenso über einen transperitonealen Zugang von vorne und über den iliosakralen Zugang von dorsal. Azetabulumfrakturen werden entweder über den ilioinguinalen oder den so genannten Kocher-Langenbeck- sowie den iliofemoralen Zugang versorgt. Gemeinsam ist all diesen Zugängen eine sehr ausgedehnte Exposition der frakturierten Knochen und damit verbunden ein ausgeprägtes zusätzliches operatives Weichteiltrauma.

Alternativen $\mathrm{zu}$ diesen extrem exponierenden Zugängen wären wünschenswert. Der Einsatz computergesteuerter navigierter Operationsverfahren zur gedeckten Einbringung von Schrauben bringt eine Reihe von Vorteilen mit sich:

- Schonung des Weichteilmantels

- Respektierung der biologischen Potenz des Frakturhämatoms

- erhöhte Präzision der Implantateinbringung

- Verhinderung von Stabilisierungen in Fehlstellung

- Reduktion von iatrogener, zugangsbedingter Morbidität
- Minimierung der Strahlenbelastung für Patient und Operationsteam

\section{Eigene Erfahrungen}

\section{Patienten}

Zwischen dem 01.01.2004 (Halle) bzw. 01.06.2004 (Jena) und dem 31.12.2005 wurden an den Kliniken für Unfall-, Handund Wiederherstellungschirurgie der BGUnfallklinik Bergmannstrost in Halle, Saale, und der Friedrich-Schiller-Universität in Jena 263 Patienten mit Beckenringverletzungen und 165 Patienten mit Azetabulumfrakturen behandelt. Dabei wurden insgesamt 98 Schrauben in navigierter Technik implantiert. Zur Stabilisierung von Beckenringfrakturen wurden 34 Iliosakralverschraubungen, 36 Schambeinastschrauben und 6 transiliakale Verschraubungen vorgenommen. Zur Stabilisierung von Azetabulumfrakturen wurden 13 supraazetabulare Schrauben, 5 Schrauben für vordere und 4 Schrauben für hintere Pfeilerfrakturen navigiert implantiert.

\section{Erreichte Verbesserungen gegenüber bisherigen Verfahren}

Neben der Schonung des Weichteilmantels und der Reduktion der zugangsbedingten Morbidität erwies sich die erhöhte Präzision der Implantateinbringung als entscheidender Vorteil. An 3 topographischen Abschnitten des Beckenrings steht für die einzubringenden Schrauben nur ein sehr schmaler Korridor zur Ver- fügung: im Bereich der iliosakralen Verschraubung, am vorderen Schambeinast und am Azetabulum. Bei der iliosakralen Verschraubung bleibt nur ein wenige $\mathrm{cm}^{2}$ großer Bereich für das sichere Einbringen von 2 Schrauben, die allerdings für die notwendige mechanische Stabilität des hinteren Beckenringes unverzichtbar sind $[1,7]$ (• Abb. 1). Auch im Bereich des vorderen Schambeinasts und über dem Azetabulum ist der für eine Schraubenplatzierung verbleibende Korridor sehr eng bemessen [6].

Die Durchleuchtungszeiten für die präoperative Akquisition des Datensatzes schwankten pro Schraube zwischen 3 und $22 \mathrm{~s}$ und betrugen im Mittel $6 \mathrm{~s}$. Entsprechende Vergleichszahlen in der Literatur ergeben für iliosakrale Schraubeneinbringungen Zeiten im Schnitt zwischen 1 min $40 \mathrm{~s}$ [2] und 2 min $6 \mathrm{~s}$ [4] und für transpubische Verschraubungen $3 \mathrm{~min}$ und $20 \mathrm{~s}$ [5]. Vor dem Hintergrund der Tatsache, dass eine Durchleuchtungszeit von 1 min am Becken einer Strahlenbelastung von $40 \mathrm{mSv}$ und damit 250 Röntgenthoraxaufnahmen oder einer CT-Untersuchung des Beckens entspricht [2], wird die Bedeutung der Verringerung der Strahlenbelastung für den Patienten im ganz besonderen Maß und darüber hinaus für das Operationsteam erkennbar.

\section{Technische Anforderungen}

Navigationsgestützte Operationsverfahren basieren auf unterschiedlichen technischen Voraussetzungen. Neben CT-ba- 
sierten Systemen mit prä- bzw. intraoperativ akquirierten Datensätzen basieren einige Verfahren auf der 3D-Fluoroskopie bzw. auf der Fluoroskopie mit einzelnen festen Durchleuchtungsbildern.

Neben der Navigation von Instrumenten und Implantaten besteht teilweise auch bereits die Möglichkeit zur navigierten Reposition von Fragmentanteilen.

Wir verwenden ein Navigationssystem $\left(\right.$ BrainLab $\left.^{\circ}\right)$, dessen Datenakquisition sich auf 3 möglichst senkrecht aufeinander stehende Durchleuchtungsbilder stützt. Dabei kommen bei Beckenringverletzungen neben der Beckenübersicht und der seitlichen Aufnahme die Inlet-, Outlet-, Obturator-Outlet-, Ala-Inlet- und Obturator-Inlet-Projektion zur Anwendung. Bei den Azetabulumfrakturen sind dies neben Beckenübersicht und seitlicher Aufnahme die Ala-, Obturator- und Obturator-Outlet-Projektion.

$\mathrm{Zu}$ Beginn der Operation wird die Bohrhülse nach Länge, Dicke und Positionierung kalibriert. Gegenüber der Kalibrierung der Kirschner-Draht-Spitze hat die Kalibrierung der Bohrhülse den Vorteil, dass eine durch eine mögliche Verbiegung des Kirschner-Drahts im Verlauf durch das Gewebe verursachte Diskrepanz der Implantatpositionierung zwischen virtuellem und realem Raum ausgeschlossen werden kann.

\section{Kasuistik}

In $-\mathrm{Abb} .2$ und 3 sind Fallbeispiele für navigierte Beckenring- und Azetabulumverschraubungen aufgeführt.

\section{Indikationsstellung zum Einsatz der Navigation}

Der Einsatz der navigierten Operationstechnik sollte derzeit aufgrund der noch geringen Erfahrungen auf ausgewählte Fälle beschränkt bleiben. Eine kritische Indikationsstellung und sorgfältige Auswahl der geeigneten Fälle trägt auch dem entsprechenden Sicherheitsanspruch für den Patienten Rechnung. In - Tab. 1 sind die an unserem Zentrum definierten Indikationen zur navigierten Verschraubung von Beckenringverletzungen und in $\mathbf{0}$ Tab. 2 die zur navi-

Trauma Berufskrankh 2007 · 9 [Suppl 2]:S168-S171 DOI 10.1007/s10039-006-1146-7

(c) Springer Medizin Verlag 2006

\section{G. O. Hofmann $\cdot$ I. Marintschev $\cdot$ T. Mückley $\cdot$ F. Mendler Beckenring- und Azetabulumverletzungen. Stellenwert der Navigation}

\section{Zusammenfassung}

Für die definitive Versorgung von Beckenringverletzungen und Azetabulumfrakturen sind meist ausgedehnte operative Zugänge erforderlich. Sie gehen mit einer umfangreichen Exposition der frakturierten Knochen und einem ausgeprägten zusätzlichen operativen Weichteiltrauma einher. Alternativen zu diesen extrem exponierenden Zugängen wären wünschenswert. Der Einsatz computergesteuerter navigierter Operationsverfahren zur gedeckten Einbringung von Schrauben ist mit einer Reihe von Vorteilen verbunden. Wie eigene Ergebnisse zeigten, ist neben der Schonung des Weichteilmantels und der Reduktion der zugangsbedingten Morbidität die erhöhte Präzision der Implantateinbringung entscheidend. Auch die Strahlenbelastung konnte aufgrund verkürzter Durch-

leuchtungszeiten stark gesenkt werden, was nicht nur den Patienten, sondern auch dem Operationsteam zugute kommt. Der Einsatz der navigierten Operationstechnik sollte allerdings derzeit aufgrund der noch geringen Erfahrungen auf ausgewählte Fälle beschränkt bleiben. Als Nachteile der Methode sind die hohen Anschaffungs- und Wartungskosten zu sehen. Da es sich um einen hochspezialisierten Eingriff handelt, sollte er nur in entsprechenden Zentren durchgeführt werden.

\section{Schlüsselwörter}

Beckenringverletzung · Azetabulumfraktur . Computergesteuerte navigierte Operation . Präzise Implantateinbringung · Strahlenbelastung

\section{Injuries to the pelvic ring and acetabulum. Value of navigation}

\section{Abstract}

The definitive treatment of injuries to the pelvic ring and of acetabular fractures usually requires extensive surgical access. This means wide exposure of the fractured bones and marked additional soft tissue trauma caused by the surgery. Alternatives to these approaches with the extreme exposure they entail would be beneficial. The use of computer-controlled navigated operation techniques for closed introduction of screws has a number of advantages. Our own experience has shown that besides the more restricted damage to the soft tissue mantle and the lower morbidity associated with the surgical approach, the increased precision with which the implants are placed also has a distinctly positive influence. It has also been possible to reduce the radiation burden substantially because of the shorter exposure times, which is good not only for the patients but also for the surgical team. At present, because little experience is available with them, the use of surgical techniques involving computer navigation should be restricted to carefully selected cases. The high costs of acquisition and maintenance have to be seen as drawbacks to the method. As these are highly specialized interventions they should not be performed except in appropriate special centres.

\section{Keywords}

Pelvic ring injury - Acetabular fracture $\cdot$ Computer-controlled navigated operation · Precision of implant placement $\cdot$ Radiation burden 


\section{Azetabulum/Becken}
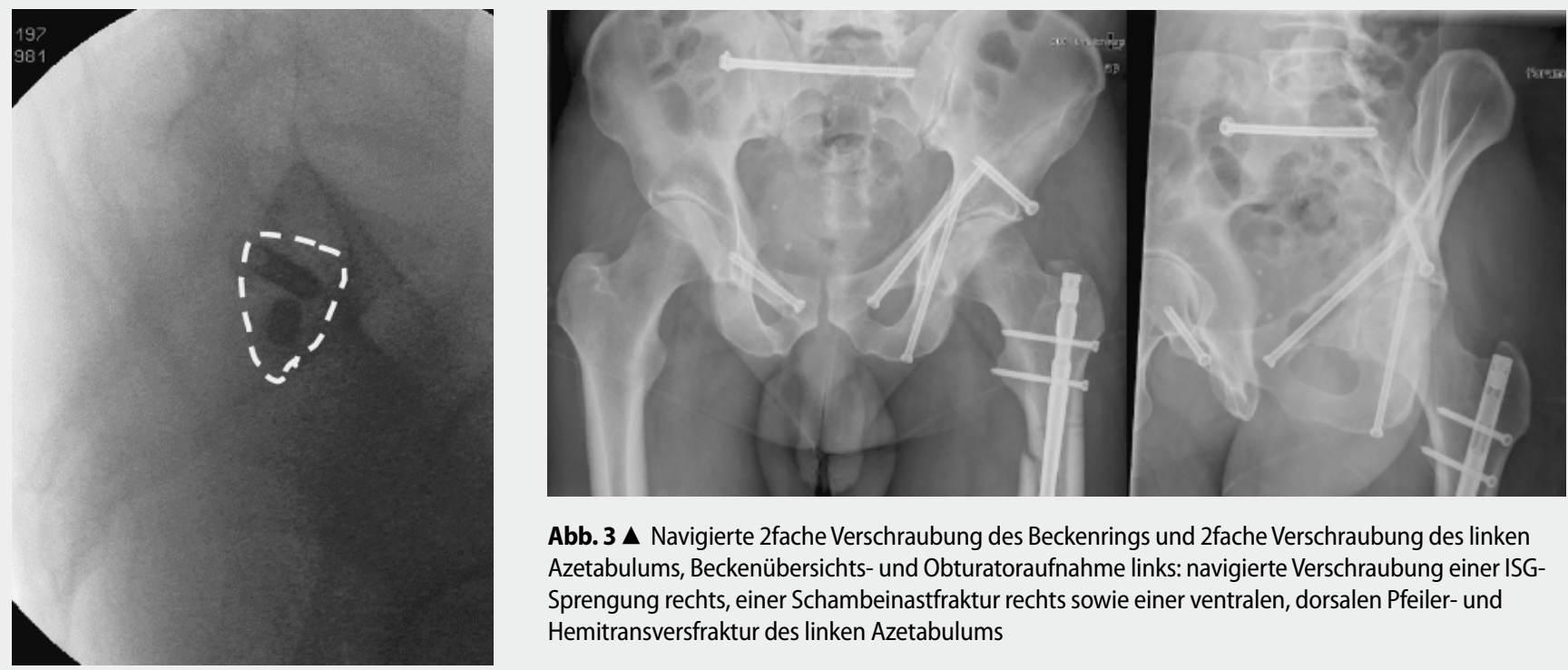

Abb. $3 \Delta$ Navigierte 2fache Verschraubung des Beckenrings und 2fache Verschraubung des linken Azetabulums, Beckenübersichts- und Obturatoraufnahme links: navigierte Verschraubung einer ISGSprengung rechts, einer Schambeinastfraktur rechts sowie einer ventralen, dorsalen Pfeiler- und Hemitransversfraktur des linken Azetabulums

Abb. $1 \Delta$ Iliosakralfuge im seitlichen Strahlengang, stabilisiert mit 2 kanülierten Schrauben
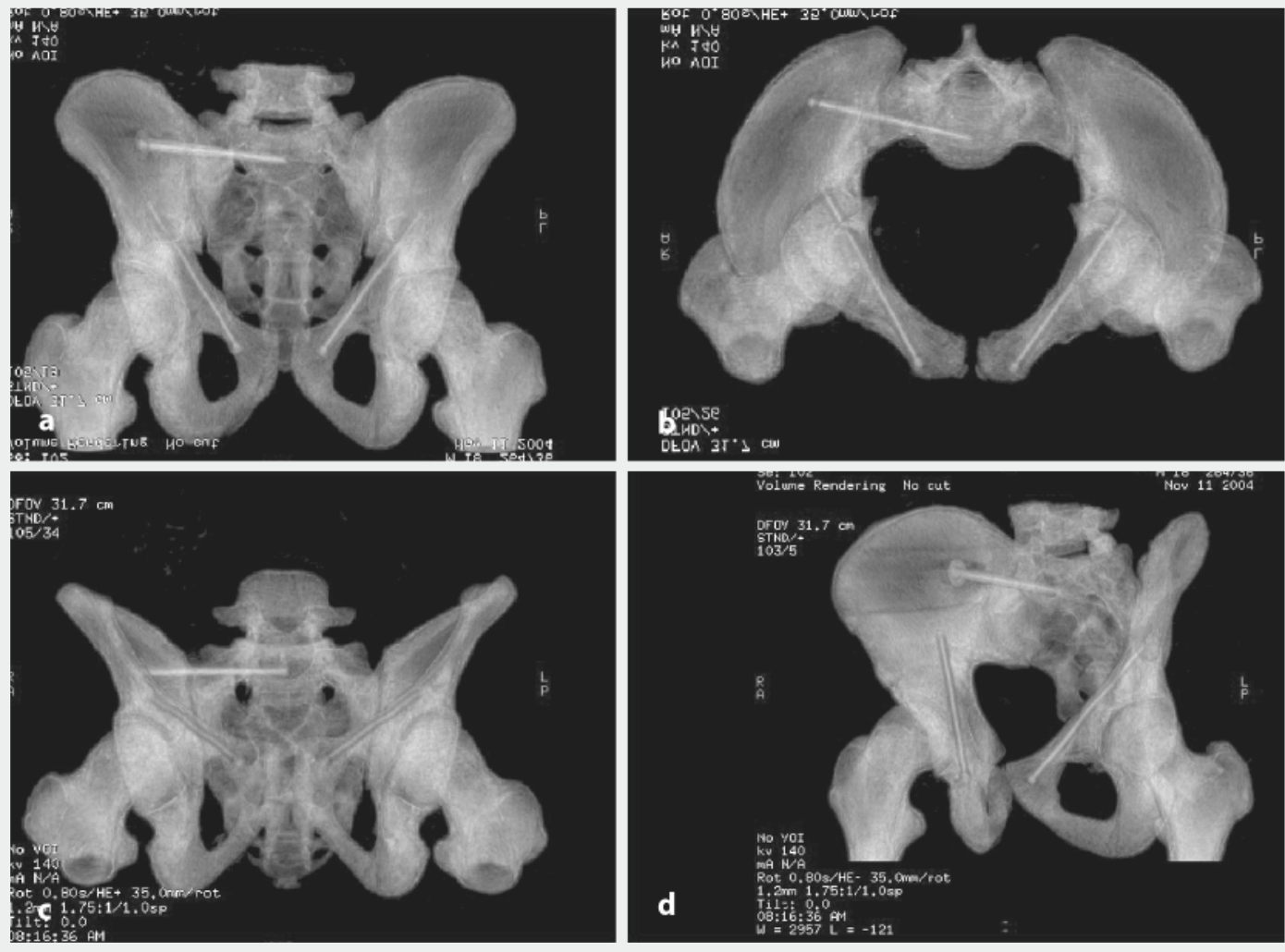

Abb. $2 \varangle$ Navigierte 3 fache Verschraubung des Beckenrings: ventrale Pfeilerfraktur links, ventrale Pfeilerfraktur rechts, ISG-Sprengung rechts, a Beckenübersichtsaufnahme, b Inlet-Aufnahme, c Outlet-Aufnahme, d Ala-Aufnahme rechts, Obturatoraufnahme links

gierten Verschraubung von Azetabulumfrakturen aufgelistet.

Kontraindikationen zum Einsatz der Navigation bei Beckenringverletzungen sind $\mathrm{u}$. E.:

- Abrissfrakturen der Spina und des Tuber ischiadicum (61A1)

- Beckenschaufelfrakturen (61A2.1, Duverney)
- Sakrumquerfrakturen $\left(61 \mathrm{~A}_{3}\right)$

- transforaminale Sakrumtrümmerfrakturen (Einklemmung der Nerven)

- weit anterior verlaufende transiliakale Luxationsfrakturen („crescent fracture“, $61 \mathrm{C} 1.2 \mathrm{a})$
Als Kontraindikationen für den Einsatz der Navigation bei Azetabulumfrakturen sehen wir an:

- jede geschlossen nicht reponierbare C-Fraktur

- Hinterwandfrakturen (62A1)

- Vorderwandfrakturen (62A3.1)

- Frakturen des hinteren Pfeilers und der Hinterwand (62A2.3) 


\begin{tabular}{|c|c|c|}
\hline $61 \mathrm{~A} 2.2$ & \multirow[t]{2}{*}{ Schambeinastfrakturen } & \multirow[t]{2}{*}{ Nur bei erheblichen Mobilisationsschmerzen } \\
\hline $61 A 2.3$ & & \\
\hline 61B1 & "open book“ & $\begin{array}{l}\text { Komplettierende ilio- oder transsakrale Verschraubung bei } \\
\text { Stabilisierung des vorderen Beckenrings }\end{array}$ \\
\hline $61 \mathrm{~B} 2$ & „lateral compression“ & $\begin{array}{l}\text { Komplettierende ilio- oder transsakrale Verschraubung, } \\
\text { Stabilisierung des vorderen Beckenrings }\end{array}$ \\
\hline 61B2.3 & Transiliakale Fraktur & 2 Schrauben transiliakal, Schambeinastverschraubung \\
\hline 61B3 & „Bilaterale B-Verletzungen“ & Ventrale und dorsale Verschraubung \\
\hline \multirow[t]{2}{*}{$61 C$} & \multirow{2}{*}{$\begin{array}{l}\text { Alle C-Verletzungen, vor- } \\
\text { ausgesetzt geschlossene } \\
\text { Reposition möglich }\end{array}$} & Dorsale und ventrale Verschraubung \\
\hline & & $\begin{array}{l}\text { Gegebenenfalls Kombination mit vertebro-pelviner Stabi- } \\
\text { lisierung im Sinne einer triangulären Osteosynthese }\end{array}$ \\
\hline
\end{tabular}

\section{Tab. 2 Azetabulumfrakturen: Indikation zur Navigation}

62A3.2 Hohe Varianten einer vorde- Transiliakale Schraube, vordere Pfeilerverschraubung ren Pfeilerfraktur

62A2 Hintere Pfeilerfraktur Verschraubung hinterer Pfeiler?

62B1 Querfrakturen Verschraubung vorderer und hinterer Pfeiler

62B2 T-Frakturen Verschraubung vorderer und hinterer Pfeiler?

62B3.2 Hoher vorderer Pfeiler, hin- Transiliakale Schraube, vordere (und hintere) Pfeilervertere Hemitransversfraktur schraubung

$62 \mathrm{C1}$ Hohe 2-Pfeiler-Fraktur, Transiliakale Schraube, vordere (und hintere) Pfeilervereinfach

\section{Literatur}

1. Ebraheim NA, Xu R, Biyani A et al. (1997) Morphologic considerations of the first sacral pedicle for iliosacral screw placement. Spine 22: 841-846

2. Hilgert RE, Finn J, Egbers HJ (2005) Technik der perkutanen SI-Verschraubung mit Unterstützung durch konventionellen C-Bogen. Unfallchirurg 108: 954-960

3. Mehlman CT, DiPasquale TG (1997) Radiation exposure to the orthopaedic surgical team during fluoroscopy: ,how far away is far enough?". J Orthop Trauma 11: 392-398

4. Nork SE, Jones CB, Harding SP et al. (2001) Percutaneous stabilization of $\mathrm{U}$-shaped sacral fractures using iliosacral screws: technique and early results. J Orthop Trauma 15: 238-246

5. Routt ML Jr, Simonian PT, Grujic L (1995) The retrograde medullary superior pubic ramus screw for the treatment of anterior pelvic ring disruptions: a new technique. J Orthop Trauma 9: 35-44

6. Shiramizu K, Naito M, Yatsunami M (2003) Quantitative anatomic characterisation of the pelvic brim to facilitate internal fixation through an anterior approach. J Orthop Surg 11: 137-140

7. Van Zwienen CMA, Van den Bosch EW, Snijders $C J$ et al. (2004) Biomechanical comparison of sacroiliac screw techniques for unstable pelvic ring fractures. J Orthop Trauma 18: 589-595
- tiefer vorderer Pfeiler und hintere Hemitransversfraktur (62 33.3$)$

- tiefe 2-Pfeiler-Frakturen $\left(62 \mathrm{C}_{2}\right)$

- 2-Pfeiler-Frakturen mit Beteiligung des Iliosakralgelenks

- intraartikuläre Fragmente oder Gelenkstufen

Insbesondere im Bereich der Belastungszone erscheinen Gelenkstufen $>1$ mm und außerhalb der Gelenkstufe $>5 \mathrm{~mm}$ als im Ergebnis nicht tolerierbar.

Besonders geeignet für die navigierte perkutane Versorgung erscheinen uns nicht dislozierte, aber instabile Frakturen (Prävention einer Sekundärdislokation) sowie Frakturen, die nach dem Zugschraubenprinzip reponierbar sind und die adäquat geschlossen reponiert werden können. Eine gute Indikation zeigt sich auch bei allen Patienten, die einen offenen Eingriff nicht tolerieren würden, oder sehr adipösen (Bevorzugung der nicht perfekten Reposition vor der $\mathrm{Zu}$ gangsmorbidität) sowie sehr alten Patienten. Hier erlaubt die In-situ-Stabilisierung eine frühzeitige Mobilisation sowie Schonung der Weichteile und der Knochensubstanz für eine verzögertprimäre Hüft-TEP.

\section{Fazit}

Die navigiert gesteuerte Implantation von Schrauben in der Chirurgie von Beckenring- und Azetabulumverletzungen erscheint ein zukunftsträchtiges Verfahren der minimalinvasiven Chirurgie. Als Nachteil ist sicher anzuführen, dass ihr Einsatz mit hohen Anschaffungs- und Wartungskosten verbunden ist. Da es sich um einen hochspezialisierten Eingriff handelt, der zudem für den Operateur mit einer flachen Lernkurve verbunden ist, erscheint uns diese Form der Chirurgie notwendigerweise als eine Zentrumsaufgabe.

\section{Korrespondierender Autor}

\section{Prof. Dr. Dr. G. O. Hofmann}

Kliniken für Unfall-, Hand- und Wiederherstellungschirurgie, BG-Kliniken Bergmannstrost, Friedrich-Schiller-Universität Jena Merseburger Straße 165, 06112 Halle, Saale gunther.hofmann@bergmannstrost.com

Interessenkonflikt. Es besteht kein Interessenkonflikt. Der korrespondierende Autor versichert, dass keine Verbindungen mit einer Firma, deren Produkt in dem Artikel genannt ist, oder einer Firma, die ein Konkurrenzprodukt vertreibt, bestehen. Die Präsentation des Themas ist unabhängig und die Darstellung der Inhalte produktneutral. 